\title{
A NOTE ON FUNCTIONS WITH DEFICIENCY SUM TWO
}

\author{
DAVID DRASIN I
}

1. Introduction. We shall apply the Ahlfors theory of covering surfaces (cf. [1], [2] and [10], Ch. XIII) to analyse functions of order $\lambda<\infty$ having

$$
\sum_{1}^{q} \delta\left(a_{i}, f\right)=2 \text {. }
$$

The problem of characterizing such functions was formally proposed by F. Nevanlinna in 1929 ([9], cf. also [6]). Nevanlinna conjectured in particular that $2 \lambda-1$ must be a positive integer and each $\delta\left(a_{i}\right)$ an integral multiple of $\lambda^{-1}$. This would imply

$$
q \leqq 2 \lambda
$$

and Weitsman's remarkable proof of (1.2) ([13]) still remains an isolated step toward resolving this hypothesis (if $f$ is entire very complete information is known [3], [4], [5], [11]).

A proof of the full F. Nevanlinna conjecture seems beyond the scope of the Ahlfors theory since this theory applies to very general exhaustions of the plane, in particular ones for which the conjecture is false.

That $f$ has finite order implies that to each $K>2$ may be associated $M<\infty$ and an unbounded $R$-set $\mathscr{P}$ where

$$
\mathscr{P}=\{R ; T(4 K R)<M T(R)\}
$$

(compare (1.3) with definition (3.3) of [7]; in [7] appear estimates on the size of $\mathscr{P})$.

In order to state our results, we recall a standard convention: if $A$ is an open set of the plane, then

$$
n(r, a, A)
$$

1 Research partially supported by National Science Foundation. 
is the cardinality of $\left\{f^{-1}(a)\right\} \cap A \cap\{|z|<r\}$, and if $\Theta_{A}(r)=$ $\left\{\vartheta ; r e^{i \vartheta} \in A\right\}$,

$$
m(r, \alpha, A)=\frac{1}{2 \pi} \int_{\Theta_{A}^{(r)}} \log \left|\frac{1}{f\left(r e^{i \vartheta}\right)-a}\right| d \vartheta .
$$

Th e or e m. Let $f$ satisfy (1.1) and be of finite order $\lambda$. Then in each disc $\{|z|<K R\} \quad(R \in \mathscr{P})$ exist unions of simply-connected components $A_{i}=A_{i}(R) \quad(1 \leqq i \leqq q)$ such that all components of $A_{i}$ meet $\{|z|=R\}$,

$$
\text { no components of } A_{i} \text { are compact subsets of }\{|z|<K R\}
$$

$$
\left|f(\zeta)-a_{i}\right|=\varepsilon \quad\left(\zeta \in \partial A_{i},|\zeta|<K R, R \in \mathscr{P}\right)
$$

and, as $R \rightarrow \infty$ in $\mathscr{P}$,

$$
\begin{gathered}
m\left(R, a_{i}, A_{i}\right) \sim \delta\left(a_{i}\right) T(R) \quad(i=1, \ldots, q) \\
n\left(K R, a_{i}, A_{i}\right)+n\left(K R, \infty, A_{i}\right)=o(1) T(R) .
\end{gathered}
$$

Conclusions (1.4) with (1.6) are implicit in [13], but (1.7), which implies that the $A_{i}$ may be viewed asymptotically as coverings of the punctured disc $\left\{0<\left|w-a_{i}\right|<\varepsilon\right\}$, is new. It is easy to see that error terms of the magnitude (1.7) can in fact occur, even in the presence of (1.1).

I thank Allen Weitsman for detecting a gap in the original version of $\S 3$.

2. Preliminary lemmas. As was noted already, we use arguments from Ahlfors's theory. Let $\mathscr{F}$ be the covering which the meromorphic function $f$ induces on the Riemann sphere and $\mathscr{F}(r)$ the covering obtained when $f$ is restricted to $\{|z|<r\}$. The natural comparison function here is $S(r)$, the mean sheet number of $\mathscr{F}(r)$ (cf. [10], p. 327), and we have the Ahlfors-Shimizu formula

$$
T(r) \sim \int_{0}^{r} S(t) t^{-1} d t
$$

For $1 \leqq i \leqq q$ and (small) $\varepsilon>0$, let $\gamma_{i}$ be the curve on the (base surface) Riemann sphere which corresponds to $\left\{\left|w-a_{i}\right|=\varepsilon\right\}$, and $D_{i}$ the interior of $\gamma_{i}$. For a fixed $r$, the mean sheet number $S\left(\gamma_{i}\right)$ of all arcs on $\mathscr{F}(=\mathscr{F}(r))^{2}$ which are over each $\gamma_{i}([10]$, p. 327) satisfies

$$
\left|S-S\left(\gamma_{i}\right)\right| \leqq h L
$$

where $h$ here and below represents a constant which depends on the $\left\{a_{i}\right\}$ and $\varepsilon$, but not on $r$ or the particular covering under consideration.

\footnotetext{
2 To limit notation, references to $r$ are often suppressed.
} 
We will need to quote the following facts later; they all require that $f$ be of finite order.

First, if $L(r)$ is the length of the relative boundary of $\mathscr{F}(r), J$. Miles has proved [8]

$$
\int_{0}^{r} L(t) t^{-1} d t=o(T(r))
$$

In addition, we shall use the elementary fact (cf. [6], p. 3) that under hypothesis (1.1)

$$
\lim _{r \rightarrow \infty} \frac{N(r, a)}{T(r)}=\left\{\begin{aligned}
1, & a \notin\left\{a_{i}\right\}, \\
1-\delta\left(a_{i}\right), & a \in\left\{a_{i}\right\} ;
\end{aligned}\right.
$$

i.e., lim sup may be replaced by limit in the definition of deficiency. Also, (1.1) implies

$$
\begin{gathered}
N\left(r, 1 / f^{\prime}\right)=o(1) T\left(r, f^{\prime}\right), \\
T\left(r, f^{\prime}\right) \sim(2-\delta(\infty, f)) T(r, f)
\end{gathered}
$$

(cf. [13], Lemma A) .

Finally, there is the following result of Collingwood-Selberg-Weitsman type (compare [12], pp. 200-201 and Lemma D of [14]).

$\mathrm{L}$ e $\mathrm{m} \mathrm{m}$ a $\mathrm{A}$. Let $f(z)$ be meromorphic with more than one deficient value, let $\mathscr{F}$ be the covering of the sphere induced by $f$, and let the $\left\{\gamma_{i}\right\},\left\{D_{i}\right\}$ be as above. For a fixed $i>0$, let $A_{i}$ be a compact portion of $\mathscr{F}$ which lies above $D_{i}$, and has the property that each point of $D_{i}$ is covered at most $p$ times in each component of $A_{i}$. Identify $A_{i}$ with its image in the $z$ plane by $f$. Then

$$
m\left(r, a_{i}, A_{i}\right) \leqq O(p) \quad(R \leqq r \leqq 2 K R, R \in \mathscr{P})
$$

where the constant implicit in (2.7) depends on $K, M$ (cf. (1.3)) and the size of two of the positive deficiencies.

3. A refinement of Ahlfors's estimates. Let $D^{i}\left(=D^{i}(r)\right)$ be the components of $\mathscr{F}(=\mathscr{F}(r))$ that lie over $D_{i}$, and introduce three subclasses $C_{i}, C_{i}^{*}, C_{i}^{* *}$ (which depend on $r$ ) as follows. The $C_{i}$ are those $D^{i}$ which are not compactly contained in $\mathscr{F}$; then those $D^{i}$ which are not contained (in the topology of $\{|z|<r\}$ ) in some $c_{j} \in C_{j}$ $(1 \leqq j \leqq q)$ are divided into $C_{i}^{*}$ (if $D^{i}$ is not simply-connected) and $C_{i}^{* *}$ otherwise.

Let $c_{i}$ be a component of $C_{i}$, let $b_{i}$ be the union of $c_{i}$ with the compact components of the complement of $c_{i}$ relative to $\mathscr{F}(r)$ and $B_{i}=B_{i}(r)$ 
the union of all the $b_{i}$. We identify the $C_{i}{ }^{*}$ and $B_{i}$ with their inverse images by $f$ in the plane.

L e m m a 1. Let $f(z)$ be meromorphic in the plane and $a_{1}, \ldots, a_{q}$ finite complex numbers. Then

$$
\begin{aligned}
\sum_{1}^{q} n\left(r, a_{i}\right) \geqq & (q-2) S(r)+n\left(r, \infty, \cup B_{i}\right)+\sum n\left(r, a_{i}, B_{i}\right) \\
& +\sum n\left(r, a_{i}, C_{i}^{*}\right)-h L(r) .
\end{aligned}
$$

Proof. Define a bordered surface $F$ by

$$
F=F(r)=\left(\bigcup_{1}^{q} B_{i}\right)^{\prime} \cap\{|z|<r\},
$$

so that

$$
\begin{aligned}
& \sum n\left(r, a_{i}\right)= \\
& \sum n\left(r, a_{i}, B_{i}\right)+\sum_{i} \sum_{j \neq i} n\left(r, a_{i}, B_{j}\right)+\sum n\left(r, a_{i}, F\right) .
\end{aligned}
$$

Rouché's theorem implies that

$$
\sum_{i} \sum_{j \neq i} n\left(r, a_{i}, B_{j}\right)=(q-1) n\left(r, \infty, \cup B_{i}\right)
$$

so (3.1) will follow at once from (3.2), (3.3) and

$$
\begin{aligned}
& \sum n\left(r, a_{i}, F\right) \geqq \\
& (q-2) S-(q-2) n\left(r, \infty, \cup B_{i}\right)+\sum n\left(r, a_{i}, C_{i}^{*}\right)-h L .
\end{aligned}
$$

To prove (3.4), let $\mathscr{F}_{1}=\mathscr{F}_{1}(r)$ be the subsurface of $\mathscr{F}$ which corresponds to $F$. Each component of $\mathscr{F}_{1}(r)$ is simply-connected and $\mathscr{F}_{1}(r) \subset \mathscr{F}_{1}\left(r^{\prime}\right) \quad\left(r<r^{\prime}\right)$; thus the $\mathscr{F}_{1}(r)$ exhaust a covering of the sphere in the sense of $[10]$, p. 341. If all $C_{i}^{*}, C_{i}^{* *}$ are now deleted from $\mathscr{F}_{1}$, we obtain $\mathscr{F}^{*}=\mathscr{F} *(r)=U F^{*}$ which now is a covering of $F_{0}$, the Riemann sphere with the $D_{i}$ deleted. It follows as in [10], pp. 342-3, that

$$
-\sum_{\mathscr{F}_{1}} \varrho\left(D^{i}\right) \equiv-\sum_{C_{i} * C_{i}^{* *}} \varrho\left(D^{i}\right) \geqq \sum_{\mathscr{F}^{*}} \varrho^{+}\left(F^{*}\right)
$$

where $\varrho$ is Euler's characteristic and $\varrho^{+}=\max (\varrho, 0)$. The right side of (3.5) is estimated by the Main Theorem ([10], p. 332):

$$
\begin{aligned}
\sum \varrho^{+}\left(F^{*}\right) & \geqq \varrho\left(F_{0}\right) S^{*}(r)-h^{*} L^{*}(r) \\
& =(q-2) S^{*}(r)-h^{*} L^{*}(r) ;
\end{aligned}
$$

here $S^{*}$ is the mean sheet number of $\mathscr{F}^{*}, h^{*}$ is a positive constant depending only on $F_{0}$ and $L^{*}$ is the length of the relative boundary of $\mathscr{F}^{*}(r)$. Clearly 


$$
L^{*} \leqq L
$$

where $L$ is as in $\S 2$, so $(3.5)-(3.7)$ yield a positive constant $h$ as in $\S 2$ with

$$
-\sum_{\mathscr{F}_{1}} \varrho\left(D^{i}\right) \geqq(q-2) S^{*}(r)-h L(r) .
$$

Let $\beta_{i}$ join $\gamma_{i}$ to $\gamma_{i+1}$ on the sphere (indexing $\bmod q$ ) and be disjoint from $\cup D_{i}$. Then if $S^{*}\left(\beta_{i}\right)$ is the mean covering number of $\beta_{i}$ in $\mathscr{F}^{*}$ we have (cf. (2.2), (3.7))

$$
\begin{aligned}
& \left|\sum S^{*}\left(\beta_{i}\right)-q S^{*}\right|<h L, \\
& \left|\sum S\left(\beta_{i}\right)-q S\right|<h L .
\end{aligned}
$$

It is straightforward from Rouché's theorem to see that if $S_{i}^{*}\left(\beta_{j}\right)$ is the mean covering number of $\beta_{j}$ from curves interior to $B_{i}$ then

and so

$$
\sum S_{i}^{*}\left(\beta_{j}\right)=q n\left(r, \infty, B_{i}\right)
$$

$$
\sum\left\{S\left(\beta_{i}\right)-S^{*}\left(\beta_{i}\right)\right\}=\sum_{i, j} S_{i}^{*}\left(\beta_{j}\right)=q n\left(r, \infty, \cup B_{i}\right) .
$$

It follows from this, (3.9) and (3.10) that

$$
\begin{aligned}
S^{*} & \geqq q^{-1} \sum S^{*}\left(\beta_{i}\right)-h L \\
& =q^{-1} \sum S\left(\beta_{i}\right)-q^{-1} \sum\left\{S\left(\beta_{i}\right)-S^{*}\left(\beta_{i}\right)\right\}-h L \\
& \geqq S-n\left(r, \infty, \cup B_{i}\right)-h L .
\end{aligned}
$$

Also $\varrho\left(D^{i}\right) \geqq 0$ unless $D^{i}$ is in $C_{i}^{* *}$. Thus (3.8) now becomes

$$
\begin{aligned}
\sum_{C_{i}^{* *}} n\left(r, a_{i}\right) & \geqq-\sum_{\mathscr{F}_{1}} \varrho\left(D^{i}\right) \\
& \geqq(q-2) S-(q-2) n\left(r, \infty, \cup B_{i}\right)-h L,
\end{aligned}
$$

and (3.4) is proved.

4. Proof of the Theorem. Choose $a_{1}, \ldots, a_{q}(q \geqq 2)$ to exhaust the deficient values of $f$; it is no loss of generality to suppose $\infty \notin\left\{a_{i}\right\}$.

Let $R \in \mathscr{P}$, and for each $i$ consider the components $D^{i}$ over $D_{i}$ in $\mathscr{F}(2 K R)$. In order to define $A_{i}$, it is first necessary to delete three subclasses of the $D^{i}$.

First, let $D_{1}^{i}$ be all compact components of $D^{i}$ in $\mathscr{F}(2 K R)$ which are simply-connected and also are $1-1$ coverings of $D_{i}$; as usual, we identify the $D_{1}{ }^{i}$ with their inverse image in the plane by $f$. It then follows from (2.7) that 


$$
m\left(r, a_{i}, D_{1}{ }^{i}\right)=O(1) \quad(R \leqq r<2 K R, R \in \mathscr{P}) .
$$

Next, let $D_{2}{ }^{i}$ be the remaining compact simply-connected components of $D^{i}$ in $\mathscr{F}(2 K R)$. Let $D_{0}$ be a component of $D_{2}{ }^{i}$. Since $D_{0}$ is compact, each $w \in D_{i}$ is covered by the same number, $p_{0}$, of times by $f$ for $z \in D_{0}$ (with due account of multiplicity). But $D_{0}$ is (connected and) simplyconnected, so $f^{\prime}$ must have $\left(p_{0}-1\right)$ zeros in $D_{0}$, and since $D_{0} \notin D_{1}{ }^{i}$, it follows that $p_{0} \geqq 1$. However (1.3), (2.5) and (2.6) readily give

$$
\begin{aligned}
& n\left(2 K R, 0,1 / f^{\prime}\right) \leqq(\log 2)^{-1} N\left(4 K R, 0,1 / f^{\prime}\right) \\
& \quad=o(1) T\left(4 K R, f^{\prime}\right)=o(1) T(4 K R, f) \\
& \quad=o(1) T(R) \quad(R \rightarrow \infty, R \in \mathscr{P}),
\end{aligned}
$$

from which we deduce that the total number of times each point $w \in D_{i}$ is covered from all $D_{2}{ }^{i}$ in $\{|z|<2 K R\}$ is $o(1) T(R)$ and, from (2.7), that

The third subclass of $D^{i}$ to be eliminated is $D_{3}{ }^{i}$; the $D^{i}$ which are compactly contained in $\mathscr{F}(2 K R)$. Thus, $D_{3}{ }^{i}$ consists of the compact components of $\mathscr{F}(2 K R)$ over $D^{i}$ which are not in $D_{1}{ }^{i}$ or $D_{2}{ }^{i}$.

$\mathrm{L}$ e $\mathrm{m} \mathrm{m}$ a 2. We have

$$
m\left(r, a_{i}, D_{3}^{i}\right)=o(1) T(R) \quad(R \leqq r<K R, R \rightarrow \infty, R \in \mathscr{P}) .
$$

Proof. Consider (3.1) for $2 K R \leqq r \leqq 4 K R$. Then the $D_{3}{ }^{i}$ are a subset of the $C_{i}^{*}(r)$ in $\mathscr{F}(r)$. When (3.1) is integrated from $2 K R$ to $4 K R,(1.1),(2.1),(2.3),(2.4)$ yield

$$
\begin{aligned}
\{(q-2)+ & o(1)\}\{T(4 K R)-T(2 K R)\} \\
\geqq & \{(q-2)+o(1)\}\{T(4 K R)-T(2 K R)\} \\
& +\log 2 \sum n\left(2 K R, a_{i}, D_{3}{ }^{i}\right) \\
& -o(1) T(4 K R) \quad(R \rightarrow \infty, R \in \mathscr{P}) .
\end{aligned}
$$

Thus

$$
\sum n\left(2 K R, a_{i}, D_{3}{ }^{i}\right)=o(1) T(R) \quad(R \rightarrow \infty, R \in \mathscr{P})
$$

and since the $D_{3}{ }^{i}$ are compact, (4.3) follows from (2.7) and (4.5).

The set $A_{i}$ demanded by the theorem consists of those components $D_{4}{ }^{i}$ of $D^{i}$ which are not in $\cup_{k=1}^{3} D_{k}{ }^{i}$ and which meet $\{|z|=R\}$, together with any compact components of their complements relative to $\{|z|<K R\}$. Thus, the $A_{i}$ are simply-connected and since no $D_{4}{ }^{i}$ is compact in $\mathscr{F}(K R),(1.4)$ and (1.5) are obvious. Now, any contribution 
to $m\left(R, a_{i}\right)$ must come from $D^{i}$ which meet $\{|z|=R\}$, but the contribution from those $D^{i}$ which meet $\{|z|=R\}$ but are not in the $D_{4}{ }^{i}$ satisfies (4.1) - (4.3). Thus

(4.6) $m\left(R, a_{i}, D_{4}^{i}\right) \sim m\left(R, a_{i}, \stackrel{4}{\cup} D_{k}^{i}\right) \sim \delta\left(a_{i}\right) T(R) \quad(R \rightarrow \infty, R \in \mathscr{P})$.

In $A_{i}-\cup_{1}^{4} D_{k}^{i},\left|f-a_{i}\right|>\varepsilon$, so the assumption that $\delta(\infty, f)=0$ with (2.4) gives that $\left|m\left(R, a_{i}, A_{i}-\cup_{1}^{4} D_{k}^{i}\right)\right|=o(1) T(R)$ which with (4.6) yields (1.6).

Finally, (1.7) is obtained from (3.1) as in the proof of (4.5) since now $A_{i} \subset B_{i}=B_{i}(r)$ for all $K R<r<2 K R$.

\section{References}

[1] AhLFORS, L.: Zur Theorie der Überlagerungsflächen. - Acta Math. 65, 1935, $157-194$.

[2] -»- Über die Anwendung differentialgeometrischer Methoden zur Untersuchung von Überlagerungsflächen. - Acta Soc. Sci. Fenn. (Nova Ser.) A. 2:6, 1937 [1938].

[3] EDREI, A., and W. H. J. Fuchs: On the growth of meromorphic functions with several deficient values. - Trans. Amer. Math. Soc. 93, 1959, $292-328$.

[4] - - Valeurs déficientes et valeurs asymptotiques des fonctions méromorphes. - Comment. Math. Helv. 33, 1959, 258-295.

[5] Fuchs, W. H. J.: Théorie de l'approximation des fonctions d'une variable complexe. - Séminaire de mathématiques superieures 26 (Été, 1967). Les Presses de l’Université de Montréal, Montréal (Québec), 1968.

[6] -»- Topics in Nevanlinna theory. - Proceedings of the NRL conference on classical function theory. Mathematics Research Center, Naval Research Laboratory, Washington, D.C., 1970, 1-32.

[7] Hayman, W. K.: On the characteristic of functions meromorphic in the plane and of their integrals. - Papers presented to J. E. Littlewood on his 80th birthday. Proc. London Math. Soc. (3) 14 A, 1965, 93-128.

[8] MrLes, J.: A note on Ahlfors' theory of covering surfaces. - Proc. Amer. Math. Soc. $21,1969,30-32$.

[9] Nevanlinna, F.: Über eine Klasse meromorpher Funktionen. - Den syvende skandinaviske matematikerkongress i Oslo 19-22 august 1929. Comptes rendus de septieme congrès des mathématiciens scandinaves tenu a Oslo 19-22 août 1929. A. W. Brøggers boktrykkeri A/S, Oslo, 1930, $81-83$.

[10] Nevanlinna, R.: Analytic functions. - Die Grundlehren der mathematischen Wissenschaften 162, Springer-Verlag, Berlin - Heidelberg - New York, 1970 .

[11] PFluger, A.: Zur Defektrelation ganzer Funktionen endlicher Ordnung. Comment. Math. Helv. 19, 1946, 91-104.

[12] TsujI, M.: Potential theory in modern function theory. - Maruzen Co., Ltd., Tokyo, 1959. 
[13] Weirsman, A.: Meromorphic functions with maximal deficiency sum and a conjecture of F. Nevanlinna. - Acta Math. 123, 1969, 115-139.

[14] - - A theorem on Nevanlinna deficiencies. - Acta Math. 128, 1972, 41-52.

\section{Purdue University}

Department of Mathematics

West Lafayette, Indiana 47907

USA

Received 6 October 1975 